\title{
ANÁLISE COMPARATIVA DA FLORÍSTICA E FITOSSOCIOLOGIA DA VEGETAÇÃO ARBÓREA DO CERRADO SENSU STRICTO NA CHAPADA PRATINHA, DF- BRASIL
}

\author{
Jeanini Maria Felfili ${ }^{1}$ \\ Manoel Cláudio da Silva Jr. ${ }^{1}$ \\ Alba Valéria Rezende ${ }^{1}$ \\ José Wagner B. Machado ${ }^{1}$ \\ Bruno M. Teles Walter ${ }^{2}$ \\ Paulo Ernane N. da Silva ${ }^{3}$ \\ John Duvall Hay ${ }^{4}$
}

Recebido em 16-01-92. Aceito em 20-12-92.

RESUMO: Foram selecionadas seis áreas de estudo, Estaçāo Ecológica de Águas Emendadas-DF, Parque Nacional de Brasília-DF, APA Gama-Cabeça do Veado-DF, Silvânia-GO, Paracatu-MG e Patrocínio-MG na Chapada Pratinha $\left(15-20^{\circ} \mathrm{S}\right.$ e $46-49^{\circ}$ W), três delas em unidades de conservação e três fora delas. Em cada área foram amostradas 10 parcelas de $1000 \mathrm{~m}^{2}(20 \times 50 \mathrm{~m})$. Todas as árvores a partir de $5 \mathrm{~cm}$ de diâmetro na base do tronco foram medidas e identificadas. A florística e a fitossociologia de cada área foi analisada e estas foram comparadas entre si pelos índices de similaridade de Sørense e de Morisita. No total foram encontradas 139 espécies e 42 famílias sendo que apenas 22 espécies foram comuns a todas as áreas. Leguminosae e Vochysiaceae apresentaram valores de importância elevados em todas as áreas. As densidades por ha variaram de 664 a 1396. Os índices de diversidade de Shannon variaram de 3,11 a 3.56. As similaridades foram altas pelo índices de Sorensen, de 0,5 a 0,7 , porém baixas pelo de Morisita quando se comparou as áreas de Paracatu e Patrocínio com as demais áreas. Foi concluído que as diferenças florísticas e principalmente estruturais foram elevadas entre estas duas áreas que são mais distantes e se situam a menores altitudes do que aquelas situadas nas unidades de conservação existentes na chapada sendo sugerida a criaçāo de novas unidades nestes Municípios.

1 - Universidade de Brasília, Departamento de Engenharia Florestal, CP 152807 CEP 70919-970, Brasília-DF.

2 - EMBRAPA/CENARGEN - SAIN - Parque Rural - CP 02372, Brasília-DF. 3 - Pesquisador do projeto Biogeografia do Bioma dos Cerrados, hoje estudante de mestrado do Departamento de Ecologia da UnB.

4 - Universidade de Brasília, Departamento de Ecologia, CP 04631, CEP 70919-970, Brasília-DF. 
Palavras chaves: florística, fitossociologia, árvores, cerrado.

\begin{abstract}
Six sites were surveyed at Chapada Pratinha in Brazil $\left(15-20^{\circ} \mathrm{S}\right.$ e $46-$ $\left.49^{\circ} \mathrm{W}\right)$. Three of them were localized in conservation units in the Federal District and the others were in Silvânia-GO, Paracatu-MG e Patrocínio-MG. Ten plots of $1000 \mathrm{~m}^{2}$ $(20 \times 50 \mathrm{~m})$ were sampled in each site. The minimum diameter for measurement of the woody plants was $5 \mathrm{~cm}$ at $0.30 \mathrm{~m}$ from the ground level. Floristic composition, importance value index and Shannon's diversity index were analyzed for each area. SØrensen's and Morisita's similarity indices were used to compare the six sites. There were 139 species belonging to 42 families. Of these, only 22 species were common to all areas. Only Leguminosae and Vochysiaceae had high importance values for all areas. The number of trees/ha ranged from 664 to 1396 . Shannon's diversity index ranged from 3.11 to 3.56. Similarities were high for Sorensen's index but low when Paracatu and Patrocínio were compared to the other areas using Morisita's index. The existing conservation units do not include all structural and floristic diversity of the region, and therefore, the creation of new units in the last two areas is suggested.
\end{abstract}

Key-words: floristics, phytosociology, trees, cerrado, Brazil.

\title{
Introdução
}

Apesar da sua importância, poucos tem sido os trabalhos florísticos e fitossociológicos realizados nos cerrados e em muitos locais não foram feitas coletas de material botânico. Este fato, aliado à relativamente pequena área deste ecossistema teoricamente protegido em unidades de conservação legalizadas (Dias 1990), dão uma idéia dos riscos de perda de informações sobre a florística da região.

Considerando que os cerrados vêm sendo ocupados desde os anos 70, como área para expansão da fronteira agrícola e que extensões considdráveis desta vegetação vem sendo transformadas em carvão vegetal e ainda, que as matas nativas permanecem contribuindo com mais de $70 \%$ do volume anual de carvão produzido no país (Abracave 1988), torna-se fácil a previsão do seu futuro.

Seriam aquelas área já "protegidas" suficientes para a conservação da variabilidade genética disponível neste ambiente? Certamente não. Quais seriam as áreas prioritárias para a implantação de novas unidades de conservação?

Com o objetivo de oferecer subsídios para o planejamento de sistemas de unidades de conservação que protejam a máxima diversidade genética em cada região, o Projeto Biogeografia do Bioma dos Cerrados iniciou seus trabalhos na Chapada Pratinha tendo sido estudadas áreas situadas em unidades de conservação (Área de Proteção Ambiental (APA) Gama Cabeça do Veado/DF, Parque Nacional de Brasília/DF, Estação Ecológica de Águas Emendadas - EEAE/DF) e fora delas, nos municípios de Paracatu/MG, Patrocínio/MG e Silvânia/GO.

Três fitofisionomias foram estudadas em todas estas áreas, a saber: cerrado (sensu stricto), cerradão (Eiten 1978, 1984) e mata de galeria não inundada 
(Ribeiro et al. 1983). Neste trabalho, será apresentada a análise florística e fitossociológica para o cerrado (sensu stricto).

\section{Material e Métodos}

Para efeito do Projeto Biogeografia do Bioma do Cerrado foram consideras as unidades fisiográficas do Brasil Central, determinadas pelo zoneamento dos sistemas de terra da América Tropical CIAT/EMBRAPA-CPAC (Cochrane, et al. 1985). Neste trabalho, foram identificados 25 sistemas de terra. Dentre estes, numa primeira etapa, foi efetuado o estudo dos recursos bióticos das "Terras altas da superfície Pratinha" e das "Terras com erosão da superfície Pratinha" que juntas formam a Chapada Pratinha.

Descrição Geral da Área

A Chapada Pratinha está situada nos estados de Minas Gerais, Goiás e Distrito Federal, com seus limites entre as latitudes $15^{\circ}$ a $20^{\circ} \mathrm{S}$ e longitudes $46^{\circ}$ a $49^{\circ} \mathrm{W}$. A altitude média da chapada está em torno de 1000 metros, com solos bem drenados, vegetação típica de savana, clima Aw segundo a classificação de Köppen, precipitação média anual em torno de $1600 \mathrm{~mm}$ e temperatura média anual de $20^{\circ}$ C. A forma predominante do relevo é de planalto, cortado por vales com declividade média inferior a $30 \%$.

Inicialmente foi realizada uma expedição exploratória por diversos municípios que fazem parte do sistema de terras da Chapada Pratinha onde foram selecionadas áreas para a efetivação do levantamento dos recursos bióticos, veja Figura 1.

\section{Descrição das Áreas Amostrais}

1 - Parque Nacional de Brasília (PNB)/DF.

Área de 30.000 ha, localizada nas coordenadas $15^{\circ} 37^{\prime}$ a $15^{\circ} 45^{\prime} \mathrm{S}$ e $47^{\circ} 54^{\prime}$ a $47^{\circ} 59^{\prime} \mathrm{W}$, com altitude média de 1100 metros, solos bem drenados, porém com grande quantidade de matas de galeria inundadas. Vegetação com predomínio do cerrado s.s..

2 - Área de Proteção Ambiental Gama-Cabeça do Veado (APA)/DF.

Compreende a Fazenda Água Limpa da Universidac̀e de Brasília, Reserva Ecológica do IBGE e Jardim Botânico de Brasília - Distrito Federal. Esta possui uma área de cerca de 9.000 ha, localiza-se entre as coordenadas de $15^{\circ} 52^{\prime} \mathrm{e}$ $15^{\circ} 59^{\prime} \mathrm{S}$ e $47^{\circ} 50^{\prime}$ a $47^{\circ} 58^{\prime} \mathrm{W}$; a altitude média é de 1.100 metros. As coordenadas das sub-áreas estudadas foram: Fazenda Água Limpa (entre $15^{\circ} 56^{\prime} \mathrm{S}$ a $15^{\circ} 59^{\prime} \mathrm{e}$ $47^{\circ} 55^{\prime}$ a $\left.47^{\circ} 58^{\prime} \mathrm{W}\right)$; Reserva Ecológica do IBGE $\left(15^{\circ} 55^{\prime}\right.$ a $15^{\circ} 58^{\prime} \mathrm{S}, 47^{\circ} 52^{\prime}$ a $47^{\circ} 55^{\prime} \mathrm{W}$ ) e Jardim Botânico de Brasília (entre $15^{\circ} 52^{\prime}$ a $15^{\circ} 54^{\prime} \mathrm{S}$ e $47^{\circ} 50^{\prime}$ a 


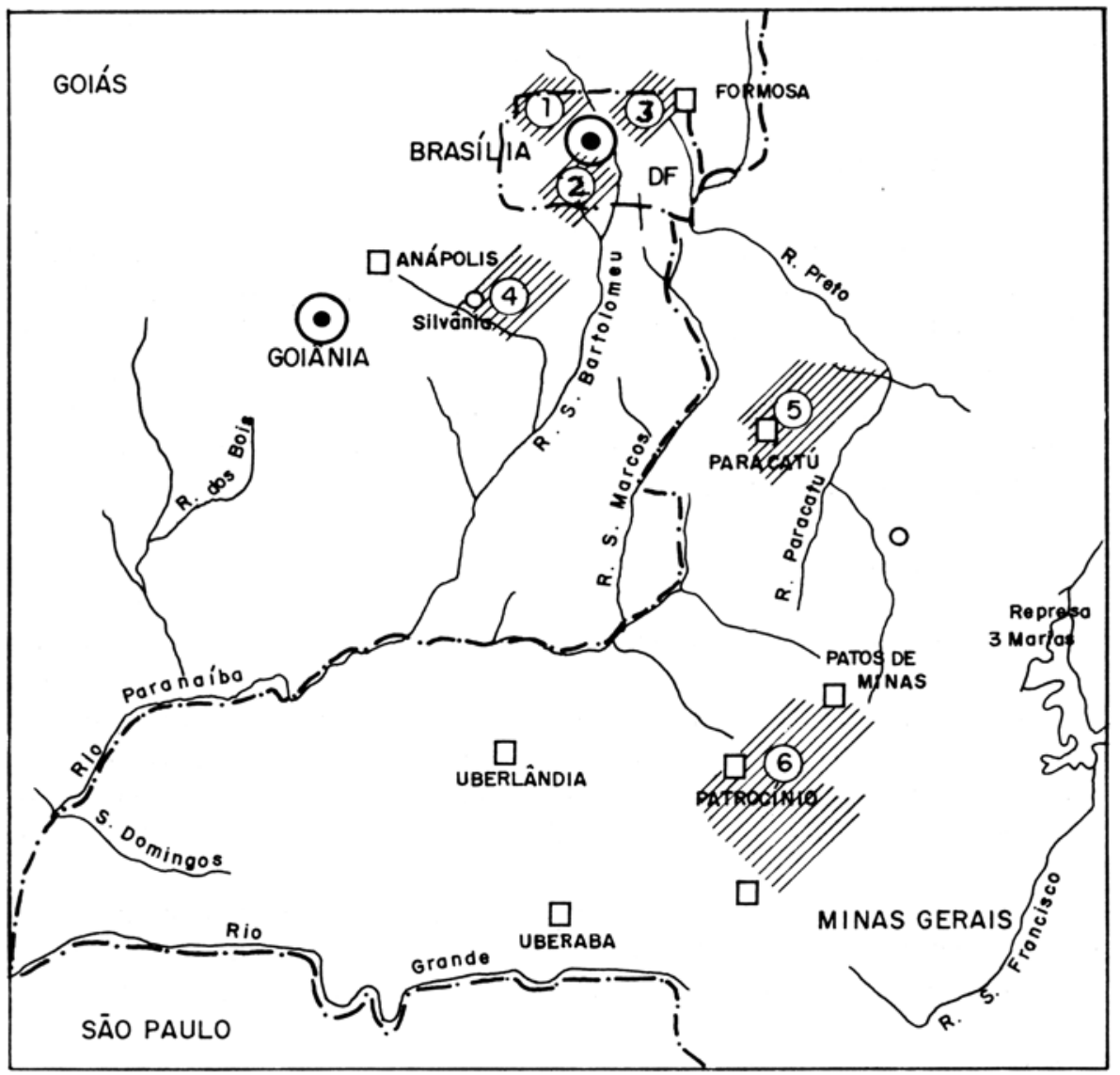

Figura 1 - Áreas estudadas na Chapada Pratinha, Brasil. (1 - Parque Nacional de Brasília (PNB)/DF, 2 - Área de Proteção Ambiental Gama-Cabeça do Veado (APA)/DF, 3 - Estação Ecológica de Águas Emendadas (EEAE)/DF. 4 - Silvânia (GO), 5 - Paracatu (MG), 6 - Patrocínio (MG)).

$\left.15^{\circ} 52^{\prime} \mathrm{W}\right)$. Vegetação com predomínio de cerrado s.s. apresentando também, extensas áreas de campo sujo e campo limpo.

3 - Estação Ecológica de Águas Emendadas (EEAE)/DF

Área de 10.000 ha., localiza-se nas coordenadas $15^{\circ} 31^{\prime}$ a $15^{\circ} 35^{\prime} \mathrm{S}$ e $47^{\circ} 32^{\prime}$ a $47^{\circ} 37^{\prime} \mathrm{W}$, com altitude média em torno de $1.100 \mathrm{~m}$, apresentando boa porcentagem de sua área coberta por vereda. A vegetação predominante é o cerrado s.s.. 
4 - Silvânia - GO

Foram estabelecidas parcelas nos municípios de Leopoldo de Bulhões, Silvânia e Vianópolis. A altitude média desta região está em torno de $1.050 \mathrm{~m}$. As amostragens foram realizadas entre as coordenadas, $16^{\circ} 30^{\prime}$ a $16^{\circ} 50^{\prime} \mathrm{S}$ e $48^{\circ} 30^{\prime}$ a $48^{\circ} 46^{\prime} \mathrm{W}$. Extensos plantios de soja estão sendo implantados na região, assim como muitas áreas com pastagens que vem ocupando o lugar dos cerrados. A vegetação predominante é o cerrado s.s. porém, com áreas extensas de cerradão.

\section{5 - Paracatu - MG}

Todas as parcelas desta área amostral foram estabelecidas neste município. A altitude média dos locais onde as parcelas foram alocadas foi de $900 \mathrm{~m}$. As coordenadas da área amostrada foram $17^{\circ} 00^{\prime}$ a $17^{\circ} 20^{\prime} \mathrm{S}$ e $46^{\circ} 45^{\prime}$ a $47^{\circ} 07^{\prime} \mathrm{W}$. Na área existem grandes plantios de soja, milho e outros cultivos. Nesta região se encontram grandes propriedades, como a da Cooperativa Agrícola de Cotia e os Projetos Mundo Novo, alguns financiados pelo governo japonês.

\section{6 - Patrocínio - MG}

O Município de Patrocínio foi base para as amostragens. As parcelas foram estabelecidas nos municípios de Patrocínio, Ibiá e Pratinha. Dentre as áreas amostradas, foi onde se notou maior ação antrópica, com grandes áreas cultivadas, principalmente com café e soja. Muitas propriedades são minifúndios intensivamente cultivados. Esta foi a área de maior extensão (coordenadas: $18^{\circ} 47^{\prime}$ a $19^{\circ} 45^{\prime} \mathrm{S}$ e $46^{\circ} 25^{\prime}$ a $47^{\circ} 09^{\prime} \mathrm{W}$ ), devido principalmente à dificuldade de se encontra rem áreas amostrais representativas. A altitude média da região é de $950 \mathrm{~m}$. No município de Patrocínio (MG), a vegetação predominante é o cerradão. O cerrado s.s. predomina no município de Pratinha (MG).

Os solos são tipicamente distróficos em todas as áreas, conforme análises efetuadas nas amostras coletadas nas parcelas amostrais pelo Prof. Dr. M. Haridasan cujos resultados serão publicados pelo mesmo em futuro próximo.

\section{Amostragem}

A vegetação arbórea foi amostrada aleatoriamente (Freese 1962). Para o cerrado s.s. foram amostradas parcelas de $20 \times 50 \mathrm{~m}\left(1000 \mathrm{~m}^{2}\right)$. Nestas parcelas foram medidos os diâmetros a $0,30 \mathrm{~m}$ do solo de todos os indivíduos que apresentaram um minímo de $5 \mathrm{~cm}$. Outras informações relevantes como por exemplo, histórico de ocorrência de foto foram registradas. Nesta fitofisionomia foram alocadas 10 parcelas por área amostral, totalizando um hectare efetivamente amostrado em cada uma delas.

Além da amostragem propiamente dita, com identificação in loco das espécies quando possível, foram efetuada incursões de coleta nas áreas amostradas e em áreas próximas para ampliar o levantamento florístico. Essas incursões foram realizadas em três diferentes épocas do ano visando encontrar o máximo de material reprodutivo. 
Todo o material coletado foi identificado por comparação no herbário da Reserva Ecológica do Instituto Brasileiro de Geografia e Estatística (UB) em Brasília e duplicatas foram enviadas para especialistas de diversas instituições nacionais e estrangeiras para completa identificação.

Os parâmetros fitossociológicos (Mueller-Dubois \& Ellenberg 1974) foram calculados de acordo com as seguintes fórmulas:

\section{Abundância}

Considera a quantidade de indivíduos de cada espécie.

A.abs = Abundância absoluta $=\mathrm{n} / \mathrm{ha}$

$\mathrm{n} / \mathrm{ha}$

A.rel. $=$ Abundância relativa $(\%)=-\times 100$

$\mathrm{n} / \mathrm{ha}$

onde,

n=número de árvores por espécie; N=Número total de árvores.

Dominância

Considera a área basal de cada espécie.

D.abs=dominância $a b s o l u t a=g / h a$

$\mathrm{g} / \mathrm{ha}$

D.rel=dominância relativa $(\%)=-\mathrm{x} 100$

$\mathrm{g} / \mathrm{ha}$

onde,

$\mathrm{g}=\pi / 4 \times \mathrm{D} 2$

$\mathrm{G}=\boldsymbol{\Sigma} \mathrm{g}$

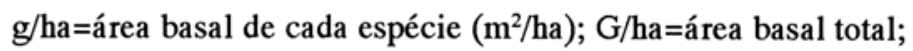

$\mathrm{D}=$ diâmetro do tronco $(\mathrm{cm})$.

3. Frequência

Considera a ocorrência das espécies nas parcelas amostradas.

F.abs=Frequência absoiluta da espécie $(\%)=\%$ de parcelas em que ocorre uma espécie;

F.abs

F.rel=Frequência relativa da espécie $(\%)=------x 100$

$\boldsymbol{\Sigma}$ F.abs 
Índice de valor de Importancia (IVI).

Reflete o grau de importância ecológica da espécie em determinado local. IVI=A.rel+D.rel+F.rel

Após o cáuculo destes parâmetros foram feitas comparações quanto a composição florística das áreas.

Análise da diversidade:

Para avaliar a diversidade florística entre as comunidades e para fazer comparações entre elas foi utilizado o índice varia de 0 a um valor positivo máximo correspondentea $\log \mathrm{S}$ e é determinado pelo número de espécies presentes na comunidade e pela base da escala logarítmica escolhida. As unidades de medida são relacionadas ao tipo de logarítmo usado no cálculo, estas são nats para loge, bits para $\log 2$ e bel para $\log 10$. Estes termos não tem sido muito utilizados atualmente, sendo mais comum expressar o tipo de logarítmo usado (Margurram 1988).

$\mathrm{H}^{\prime}=-\boldsymbol{\Sigma}$ piln pi

onde,

H'=Índice de diversidade de Shannon;

$\mathrm{pi}=\mathrm{ni} / \mathrm{N}$

ln= Logritmo neperiano; ni=número de indivíduos amostrados da espécie $\mathrm{i}$;

$\mathrm{N}=$ número total de indivíduos amostrados; $\mathrm{S}=$ número de espécies amostradas.

Análise da similaridade

É usada para comparar a composição específica de duas comunidades, ou amostras dentro da mesma comunidade. Existem comparações qualitativas, baseadas na presença e ausência das espécies e quantitativas, baseadas na abundância das espécies (Horn 1966).

Índice de Sorensen (qualitativa) - varia de 0 a 1.

2c

$\mathrm{CCs}=$

$a+b$

onde, 
CCs=Índice de Sorensen

a =número de espécies na comunidade $1 ; \mathrm{b}=$ número de espécies na comunidade 2 ; $\mathrm{c}=$ número de espécies comuns às duas comunidades.

Índice de Morisita (quantitativa) - varia de 0 a 1.

$\mathrm{Cn}=2 \Sigma \mathrm{Xi} . \mathrm{Yi} /(\mathrm{A}+\mathrm{B}) \mathrm{Nx} \cdot \mathrm{Ny}$

onde,

$\mathrm{A}=\mathrm{Xi}(\mathrm{Xi}-1) / \mathrm{Nx}(\mathrm{Nx}-1)$

$\mathrm{B}=\mathrm{Yi}(\mathrm{Yi}-1) / \mathrm{Ny}(\mathrm{Ny}-1)$

$\mathrm{Xi}=$ número de indivíduos da espécie $\mathrm{i}$ da comunidade $\mathrm{x}$; $\mathrm{Y} \mathrm{i}=$ número de indivíduos da espécie i na comunidade $\mathrm{y} ; \mathrm{Nx}=$ número total de indivíduos na comunidade $\mathrm{x}$; $\mathrm{Ny}=$ número total de indivíduos na comunidade $\mathrm{y}$;

Como regra geral, para ambos os índices, uma similaridade maior que 0,5 é considerada alta. E, quanto mais próximo o valor de 1 , maior a similaridade.

\section{Resultados e Discussão}

\section{Composição florística}

Nas seis áreas amostradas no cerrado s.s. da Chapada Pratinha foram encontradas 139 espécies distribuidas em $\mathbf{4 2}$ famílias. O número de espécies e famílias por área foi respectivamente:Estação Ecológica de Aguas Emendadas, 31 famílias e 72 espécies; APA Gama-Cabeça do veado 31 familias e 66 espécies; no Parque Nacional de Brasília foram encontradas 26 famílias e 55 espécies. No município de Paracatu, 33 famílias e 60 espécies, em Patrocínio 32 famílias e 68 espécies e em Silvânia 31 famílias e 68 espécies (Tabela 1 ).

Cada área apresentou uma combinação de menos de 80 espécies e de aproximadamente 30 famílias.

Somente 22 espécies pertencentes a 14 famílias foram comuns a todas as áreas amostradas: Apocynaceae (Aspidosperma tomentosum), Araliaceae (Didymopanax macrocarpum), Bignoniaceae (Tabebuia ochracea, T. caraiba), Caryocaraceae (Caryocar brasiliense), Compositae (Eremanthus glomerulatus, Piptocarpa rotundifolia), Connraceae (Connarus suberosus), Erythoxylaceae (Erythoxylum suberosum, E. tortuosum), Guttiferae (Kielmeyera coriacea), Leguminosae (Acosmium dasycarpum, Bowdichia virgiliodes, Dimorphandra mollis, Machaerium opacum, Stryphnodendron adsringens), Malpighiaceae (Byrsonima coccolobifolia, B. verbascifolia), Ochnaceae (Ouratea hexasperma), Proteaceae (Roupala montana), Stryracaceae (Styrax ferrugineus), Vochysiaceae (Qualea grandiflora). 
Estas podem ser consideradas como características da flora arbórea da Chapada Pratinha.

Diversidade

As áreas mais diversas conforme os índices de Shannon foram respectivamente, Estação Ecológica de Águas Emendadas (3,62), Patrocínio (3,53), APA Gama-Cabeça do Veado (3,56), Parque Nacional de Brasília (3,34), Silvânia(3,31), Paracatu $(3,11)$. Dentre as áreas não protegidas, Patrocínio apresentou maior diversidade.

Tabela 1 - Famílias e espécies encontradas no cerrado sensu stricto da Chapada Pratinha - Brasil com seus respectivos Índices de Valor de importância (IVI)

* O número de espécies por família esta entre parênteses atrás do nome da família e os valores de IVI estão abaixo do nome de cada localidade ** A posição de cada espécie na respectiva localidade de acordo com o IVI esta colocada ao lado deste entre parênteses

\begin{tabular}{|c|c|c|c|c|c|c|}
\hline Famílias/espécies & $\begin{array}{l}\text { EEAE } \\
\text { DF }\end{array}$ & $\begin{array}{l}\text { APA } \\
\text { DF }\end{array}$ & $\begin{array}{l}\text { PNB } \\
\text { DF }\end{array}$ & $\begin{array}{l}\text { PAR } \\
\text { MG }\end{array}$ & $\begin{array}{l}\text { PAT } \\
\text { MG }\end{array}$ & $\begin{array}{l}\text { SIL } \\
\text { GO }\end{array}$ \\
\hline 1. ANACARDIACEAE (5) & - & - & - & 2.39 & 0.82 & 1.33 \\
\hline 1. Anacardium sp. & - & - & - & - & - & $0.43(68)$ \\
\hline 2. Anacardium sp. & - & - & - & - & - & $0.90(55)$ \\
\hline 3. Astronium fraxinifolium Schott & - & - & - & $1.63(34)$ & - & - \\
\hline 4. Astronium sp. & - & - & - & $0.76(51)$ & - & - \\
\hline 5. Schinus sp. & $\cdot$ & - & - & - & $0.82(55)$ & - \\
\hline 2. ANNONACEAE (5) & 7.62 & - & 3.96 & 16.44 & 20.41 & 8.52 \\
\hline 6. Annona coriacea Mart. & $0.43(66)$ & - & - & $6.66(15)$ & $3.81(29)$ & $2.61(31)$ \\
\hline 7. A. crassiflora Mart. & $7.19(12)$ & -3 & $3.96(25)$ & $7.59(12)$ & $6.15(16)$ & $5.03(17)$ \\
\hline 8. Cardiopetalum calophyllum Schl & - & - & - & - & - & $0.88(57)$ \\
\hline 9. Xylopia aromatica Lam. & - & - & - & $2.19(31)$ & $1.06(53)$ & - \\
\hline 10. $X$. sericea St. Hil. & $\cdot$ & - & - & - & $9.39(8)$ & - \\
\hline 3. APOCYNACEAE (4) & 8.24 & 9.56 & 6.05 & 0.89 & 1.79 & 7.62 \\
\hline 11. Aspidosperna macrocarpon Mart. & $2.36(34)$ & $3.87(29)$ & $3.47(28)$ & - & - & $2.29(37)$ \\
\hline 12. Aspidosperma tomentosum Mart & $3.96(27)$ & $5.31(18)$ & $2.58(34)$ & $0.89(46)$ & $0.59(69)$ & $2.85(28)$ \\
\hline $\begin{array}{l}\text { 13. Hancornia speciosa Nees \& Mart. } \\
\text { 14. Himathantus obovatus (M. Arg.) }\end{array}$ & $1.92(39)$ & - & - & - & $0.61(62)$ & $1.90(42)$ \\
\hline Woods & - & $0.38(64)$ & - & - & $0.59(67)$ & $0.58(61)$ \\
\hline
\end{tabular}


Cont. Tabela 1

Famílias/espécies

EEAE APA PNB PAR PAT SIL

DF DF DF MG MG GO

4. ARALIACEAE (1)

15. Didymopanax macrocarpum

(C. \& S.) Seem.

\section{BIGNONIACEAE (4)}

16. Tabebuia caraiba (Mart.) Bureau

17. T. ochracea (Cham.) Standl.

18. Zeyhera montana Mart.

19. Cybistax anisiphylitica Mart.

$\begin{array}{rrrrrr}4.83 & 11.07 & 3.93 & 0.83 & 1.13 & 4.57 \\ 4.83(21) & 11.07(8) & 3.93(26) & 0.83(48) & 1.13(51) & 4.57(18)\end{array}$

$\begin{array}{rrrrrr}2.56 & 2.17 & 3.48 & 2.42 & 6.45 & 15.77 \\ 1.37(50) & 0.37(66) & 2.92(31) & 1.00(44) & 0.94(54) & 12.29(5) \\ 0.79(57) & 1.80(45) & 0.56(55) & 1.42(39) & 4.87(21) & 3.48(21) \\ 0.40(70) & - & - & - & - & - \\ - & - & - & - & 0.64(59) & -\end{array}$

6. BOMBACACEAE (5)

1.88

2.25

7.84

5.29

2.83

20. Eriotheca gracilipes (Schum.) Robins

21. E. pubescens (Mart. \& Zucc.)

Schot. \& Endl.

22. Pseudobombax longiflorum

(Mart. \& Zucc.) Robyns

23. P. tomentosum

(Mart. \& Zucc.) Robyns

$0.93(56) \quad 2.25(40) \quad 7.84(13) \quad 2.91(27)$

- $1.96(41)$

$0.48(63)$

- $0.44(67)$

$0.47(44)$

24. Pseudobombax sp.

7. CARYOCARACEAE (1)

25. Caryocar brasiliense Camb.

$\begin{array}{lll}12.26 & 12.46 & 15.62\end{array}$

$12.26(6) \quad 12.46(5) \quad 15.62(6)$

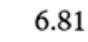

$6.81(14)$

5.07

$0.43(69)$

\section{$1.43 \quad 1.72$}

4.21

3.30

26. Austroplenkia populnea

(Reiss) Lund

$1.43(48) \quad 1.72(47)$

4.21(25)

$3.30(24)$

9. CHRYSOBALANACEAE (2)

27. Couepia grandiflora

(Mart. \& Zucc.) Benth.

28. Hirtella glandulosa Spreg.

10. COMBRETACEAE (1)

29. Terminalia argentea Mart. \& Zucc.

11. COMPOSITAE (3)

30. Eremanthus glomerulatus Less

31. Piptocarpha rotundifolia

(Less.) Bak.

32. Vernonia sp.

$\begin{array}{rrrrrr}9.96 & 11.53 & 5.76 & 34.85 & 5.13 & 4.46 \\ 4.53(23) & 7.22(11) & 1.07(49) & 1.38(41) & 0.59(66) & 2.37(36) \\ & & & & & \\ 5.43(19) & 3.55(33) & 4.69(21) & 33.47(2) & 4.54(23) & 2.09(39) \\ - & 0.76(58) & - & - & - & -\end{array}$


Cont. Tabela 1

Famílias/espécies

$$
\begin{array}{llllll}
\text { EEAE } & \text { APA } & \text { PNB } & \text { PAR } & \text { PAT } & \text { SIL } \\
\text { DF } & \text { DF } & \text { DF } & \text { MG } & \text { MG } & \text { GO }
\end{array}
$$

33. Connarus suberosus Planch.

34. Rourea induta Planch.

13. DILLENIACEAE (2)

35. Curatella americana L.

36. Davilla elliptica St. Hil

14. EBENACEAE (1)

37. Diospyros burchellii Hiem

15. ERYTHROXYLACEAE (4)

38. Erythroxylum deciduum St. Hil.

39. E. suberosum St. Hil.

40. E. tortuosum Mart.

41. Erythroxylum sp.

16. EUPHORBIACEAE (1)

42. Pera glabrata (Schott.) Baill.

17. FLACOURTIACEAE (1)

43. Casearia sylvestris $\mathrm{Sw}$.

18. GUTTIFERAE (7)

44. Kielmeyera coriacea

(Spreng.) Mart.

$45 K$. lathrophyton $\mathrm{N}$. Saddi

46. $K$. speciosa St. Hil.

47. Kielmeyera $\mathrm{sp}_{1}$

48. Kielmeyera $\mathrm{sp}_{2}$

49. Kielmeyera $\mathrm{sp}_{3}$

50. Kielmeyera $\mathrm{sp}_{4}$

19. HIPPOCRATEACEAE (1)

51. Salacia crassifolia (Mart.) Peyr.

20. ICACINACEAE (1)

52. Emmotum nitens (Benth.) Miers

21. LABIATAE (1)

53. Hyptis cana Pohl ex. Benth.

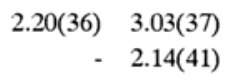

$2.20(36) \quad 3.03(37)$

- 2.14(41)

$5.15(18)$

$9.52(7)$

2.63(36)

$2.93(27)$

$\begin{array}{llll}4.20 & 2.08 & 1.76 & 43.27\end{array}$

6.28

1.98(38)

$2.22(35) \quad 2.08(43) \quad 1.76(39)$

43.27(1)

- $6.28(12)$

\section{$\begin{array}{lll}1.47 & 1.80 \quad 0.54\end{array}$}

$1.47(46) \quad 1.80(46) \quad 0.54(57)$

1.34

7.15

$1.34(48) \quad 7.15(11)$

14.1

$\begin{array}{ll}0.39(72) & 1.12(51) \\ 6.81(14) & 3.96(28)\end{array}$

6.81(14) $\quad 3.96(28) \quad 6.27(17)$

$6.94(13) \quad 2.60(38) \quad 1.53(43)$

10.53

30.06

7.64

$0.68(59)$

$2.32(38) \quad 2.22(38)$

$\begin{array}{lll}6.36(16) & 16.75(3) & 2.39(35)\end{array}$

$3.49(24)$

$2.24(40) \quad 3.03(26)$

$8.75(10)$

$$
\begin{array}{rr}
0.79 & 3.37 \\
0.79(50) & 3.37(32)
\end{array}
$$

0.41

1.40

0.72

$0.41(68)$

$1.40(40)$

$0.72(57)$

$$
23.49
$$

7.39

20.68

1.54

25.27

27.93

13.92(5)

4.28(26)

$4.24(24)$

1.54(36)

9.67(7) 26.12(3)

$0.40(69)$

$\begin{array}{rr}8.72(9) & 0.58(60) \\ - & 3.07(36)\end{array}$

$8.47(9)$

$7.97(12)$

$0.45(65)$

$1.54(36)$
$-\quad 8.96(9)$

$5.07(19) \quad 1.81(43)$

$1.57(43)$

$\begin{array}{lll}1.60 & 2.04 & 9.08\end{array}$

2.80

$1.60(42) \quad 2.04(44) \quad 9.08(8)$

- $2.80(29)$

3.51

1.30

$3.51(23)$

- $1.30(50)$

0.88

$0.88(47)$ 
Cont. Tabela 1

\begin{tabular}{|c|c|c|c|c|c|c|}
\hline Famílias/espécies & $\begin{array}{l}\text { EEAF } \\
\text { DF }\end{array}$ & $\begin{array}{l}\text { APA } \\
\text { DF }\end{array}$ & $\begin{array}{l}\text { PNB } \\
\text { DF }\end{array}$ & $\begin{array}{l}\text { PAR } \\
\text { MG }\end{array}$ & $\begin{array}{l}\text { PAT } \\
\text { MG }\end{array}$ & $\begin{array}{l}\text { SIL } \\
\text { GO }\end{array}$ \\
\hline 54. Nāo identificada & - & - & - & - & $4.50(24)$ & \\
\hline 55. Ocotea $\mathrm{sp}_{1}$ & - & $0.94(53)$ & - & - & - & - \\
\hline 56. Ocotea $\mathrm{sp}_{2}$ & - & - & - & - & $1.48(45)$ & 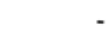 \\
\hline 23. LEGUMINOSAE (19) & 58.91 & 43.93 & 47.84 & 35.93 & 33.79 & 28.93 \\
\hline - CAESALPINOIDEAE (6) & 28.91 & 14.42 & 11.64 & 12.48 & 11.70 & 9.66 \\
\hline $\begin{array}{l}\text { 57. Acosmium dasycarpum } \\
\text { (Vog.) Yakovl. }\end{array}$ & $1.05(54)$ & $1.06(52)$ & $1.38(45)$ & $3.00(26)$ & $3.01(35)$ & $0.45(63)$ \\
\hline 58. Acosmium subelegans & & & & & & \\
\hline (Mohl) Yakovl. & - & - & - & - & $4.68(22)$ & - \\
\hline 59. Copaifera langsdorffii Desf. & - & - & $3.21(29)$ & - & - & 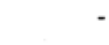 \\
\hline $\begin{array}{l}\text { 60. Hymenea stignocarpa Mart. } \\
\text { ex Hayne }\end{array}$ & $1.09(53)$ & $3.57(32)$ & $2.29(36)$ & - & $4.01(26)$ & $1.37(48)$ \\
\hline $\begin{array}{l}\text { 61. Sclerolobium aureum } \\
\text { (Tul.) Benth. }\end{array}$ & - & - & - & - & - & $6.09(13)$ \\
\hline 62. S. paniculatum Vog. & $26.77(1)$ & $9.79(9) 4$ & $1.76(20) 9.48($ & - & $1.75(44)$ & \\
\hline - MIMOSOIDEAE (6) & 17.66 & 10.16 & 19.20 & 7.84 & 4.24 & 10.46 \\
\hline 63. Cassia sp. & $6.71(15)$ & - & $0.58(53)$ & - & - & - \\
\hline 64. Dimorphandra mollis Benth. & $6.11(17)$ & $3.10(35)$ & $3.74(27)$ & $7.14(13)$ & $0.68(58)$ & $3.35(22)$ \\
\hline 65. Enterolobium ellipticum Benth. & $0.53(60)$ & $2.55(39)$ & $2.43(35)$ & - & - & $0.64(60)$ \\
\hline 66. Mimosa clausennii Benth. & - & $0.37(67)$ & $1.57(42)$ & - & - & 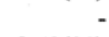 \\
\hline $\begin{array}{l}\text { 67. Plathymenia reticulada Benth. } \\
\text { 68. Stryphnodendron adstringens }\end{array}$ & - & - & $4.39(22)$ & - & - & $2.42(33)$ \\
\hline (Mart.) Cov. & $4.31(25)$ & $4.14(22)$ & $6.49(19)$ & $0.70(56)$ & $3.56(30)$ & 4.05(19) \\
\hline - PAPILIONOIDEAE (7) & 12.34 & 19.35 & 17.00 & 15.41 & 17.85 & 8.81 \\
\hline 69. Andira paniculata Benth. & - & - & - & - & - & $1.30(49)$ \\
\hline 70. Bowdichia virgilioides Kunth & $4.63(22)$ & $0.48(61)$ & $1.88(37)$ & $9.30(10)$ & $1.56(44)$ & $3.13(25)$ \\
\hline 71. Dalbergia violacea (Vog.) Malme & $2.46(34)$ & $12.07(6)$ & $10.96(7)$ & - & $12.83(5)$ & $2.05(40)$ \\
\hline 72. Machaerium acutifolium Vog. & - & $0.38(65)$ & - & - & - & $1.41(47)$ \\
\hline 73. Machaerium opacum Vog. & $0.78(58)$ & $0.80(55)$ & $2.64(33)$ & $6.31(17)$ & $3.46(31)$ & $0.92(54)$ \\
\hline $\begin{array}{l}\text { 74. Pterodon pubescens } \text { Benth. } \\
\text { 75. Vatairea macrocarpa (Benth.) }\end{array}$ & $3.99(26)$ & $4.42(24)$ & $1.52(44)$ & - & - & . \\
\hline Ducke. & $0.48(62)$ & $1.20(50)$ & - & - & - & - \\
\hline 24. LYTHRACEAE (1) & 1.35 & 0.78 & - & 3.35 & 6.45 & 10.50 \\
\hline 76. Lafoensia pacari St. Hil. & $1.35(51)$ & $0.78(59)$ & - & $3.35(35)$ & $6.45(14)$ & $10.50(6)$ \\
\hline 25. LOGANIACEAE (1) & 2.53 & 3.57 & 4.78 & - & 0.62 & 1.05 \\
\hline 77. Strychnos pseudoquina St. Hil. & $2.53(31)$ & $3.57(31)$ & $4.78(19)$ & - & $0.62(60)$ & $1.05(52)$ \\
\hline 26. MALPIGHIACEAE (8) & 22.76 & 17.06 & 18.32 & 21.12 & 13.71 & 13.57 \\
\hline 78. Byrsonima coccolobifolia Kunth. & $6.06(18)$ & $3.81(30)$ & $8.21(11)$ & $0.71(52)$ & $7.17(12)$ & $3.75(20)$ \\
\hline
\end{tabular}


Cont. Tabela 1

\begin{tabular}{l} 
Famílias/espécies \\
\hline 79. Byrsonima crassa Nied. \\
80. B. intermedia Adr. Juss. \\
81. Byrsonima ligustrifolia Adr. Juss. \\
82. Byrsonima sp. \\
83. B. verbascifolia Rich. ex \\
Adr. Juss. \\
84. Heteropteris byrsonimaefolia \\
Adr. Juss. \\
85. Heteropteris sp.
\end{tabular}

\section{MELASTOMATACEAE (7)}

86. Miconia albicans (Sw.) Triana

87. Miconia ferrugiana (DC.) Cogn.

88. Miconia pohliana Cogn.

89. Miconia sellowiana Naud.

90. Miconia sp.

91. Miconia sp2

92. Tibouchina sp.

$\begin{array}{llllll}\text { EEAE } & \text { APA } & \text { PNB } & \text { PAR } & \text { PAT } & \text { SIL } \\ \text { DF } & \text { DF } & \text { DF } & \text { MG } & \text { MG } & \text { GO }\end{array}$

$\begin{array}{rrrrrr}- & 7.85(10) & 3.18(30) & 6.31(18) & - & 6.06(14) \\ - & - & - & - & - & 0.45(64) \\ - & - & - & 5.57(19) & - & - \\ - & - & - & 3.75(22) & - & - \\ & & & & & \\ 6.56(16) & 5.40(17) & 6.93(14) & 3.80(21) & 5.95(17) & 3.31(23) \\ & & & & & \\ 7.42(11) & - & - & 1.03(43) & 0.59(68) & - \\ 2.72(30) & - & - & - & - & - \\ & & & & & \\ 4.21 & 16.39 & 2.01 & - & 2.88 & 0.49 \\ - & - & - & - & 0.61(65) & - \\ 1.30(52) & 5.27(20) & 1.22(46) & - & - & - \\ 1.38(49) & 5.02(21) & 0.79(52) & - & - & 0.49(62) \\ 0.51(61) & - & - & - & - & - \\ - & 6.10(14) & - & - & - & - \\ 1.02(55) & - & - & - & - & - \\ - & - & - & - & 2.27(39) & -\end{array}$

28. MORACEAE (1)

93. Brosimum gaudichaudii Tréc.

29. MYRSINACEAE (2)

94. Cybianthus detergens Mart.

95. Rapanea guianensis Aubl.

\section{MYRTACEAE (14)}

96. Blepharocalyx salicifolia Berg.

97. Eugenia dysenterica DC.

98. Myrcia sp.

99. Myrcia tomentosa (Aubl.) DC.

100. Myrcia variabilis DC.

101. Nāo identificada 1

102. Nāo identificada 2

103. Nāo identificada 3

104. Nāo identificada 4

105. Psidium myrsinoides Berg.

106. Psidium warmingianun Kunth

107. P.sp.1

108. P.sp. 2

109. Siphoneugena densiflora Berg.

\section{NYCTAGINACEAE (3)}

110. Guapira noxia (Netto) Lund.

111. Guapira sp.
2.22

$3.41 \quad 1.59$

$0.73(59)$

1.49(44)

$3.41(34)$

$1.59(41)$

2.16(32)

$6.20(15)$

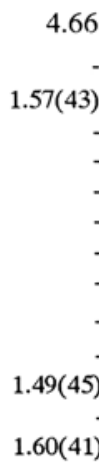

18.26

48.31

3.20

$12.15(6)$

$19.15(2)$

14.11(4)

$0.70(55)$

2.08(33)

$0.67(60)$

$3.96(27)$

1.77(41)

$0.70(57)$

1.11(48)

- 1.03(53)

$0.86(59)$

7.96(11)

$3.32(33)$

$0.44(66)$

$0.87(58)$

$$
\text { ) }
$$$$
\text { - } 3.32(33)
$$

$\begin{array}{rrrrrr}6.33 & 7.55 & 4.32 & 0.71 & 1.12 & 1.53 \\ 3.86(28) & 7.17(12) & 4.32(23) & 0.71(52) & - & 1.09(51) \\ - & - & - & - & 1.12(52) & -\end{array}$


Cont. Tabela 1

\begin{tabular}{|c|c|c|c|c|c|c|}
\hline Famílias/espécies & $\begin{array}{l}\text { EEA } \\
\text { DF }\end{array}$ & $\begin{array}{l}\text { APA } \\
\text { DF }\end{array}$ & $\begin{array}{l}\text { PNB } \\
\text { DF }\end{array}$ & $\begin{array}{l}\text { PAR } \\
\text { MG }\end{array}$ & $\begin{array}{l}\text { PAT } \\
\text { MG }\end{array}$ & $\begin{array}{l}\text { SIL } \\
\text { GO }\end{array}$ \\
\hline 112. Neea theifera Oerst. & $2.47(32)$ & $0.38(63)$ & - & - & - & $0.44(65)$ \\
\hline 32. OCHNACEAE (1) & 10.90 & 20.56 & 22.26 & 1.50 & 1.74 & 5.53 \\
\hline $\begin{array}{l}\text { 113. Ouratea hexasperma } \\
\text { (St. Hil.) Benth. }\end{array}$ & $10.90(8)$ & $20.56(1)$ & $22.26(2)$ & $1.50(38)$ & $1.74(42)$ & $5.53(15)$ \\
\hline 33. OPILIACEAE (1) & - & - & - & 0.67 & - & - \\
\hline 114. Agonandra brasiliensis Miers & - & - & - & $0.67(61)$ & - & - \\
\hline 34. PROTEACEAE (1) & 4.40 & 5.65 & 8.45 & 1.32 & 6.64 & 7.95 \\
\hline 115. Roupala montana Aubl & $4.40(24)$ & $5.65(16)$ & $8.45(10)$ & $1.32(42)$ & $6.64(13)$ & $7.95(9)$ \\
\hline 35. RUBIACEAE (3) & 4.19 & 6.09 & - & 3.99 & - & 1.42 \\
\hline $\begin{array}{l}\text { 116. Alibertia edulis (L.Rich.) A. Rich. } \\
\text { 117. Palicourea rigida Kunth } \\
\text { 118. Tocoyena formosa (C. \& S.) }\end{array}$ & $3.80(29)$ & $6.09(15)^{-}$ & - & $\begin{array}{r}3.99(20) \\
-\end{array}$ & - & $1.42(46)$ \\
\hline Schum. & $0.39(73)$ & - & - & - & - & - \\
\hline 36. SAPINDACEAE (1) & - & - & - & - & 3.01 & - \\
\hline 119. Matayba guinensis Aubl. & - & - & - & - & $3.01(34)$ & - \\
\hline 37. SAPOTACEAE (2) & 11.77 & 5.45 & 9.38 & - & - & 5.50 \\
\hline 120. Pouteria ramiflora (Mart.) Radlk. & $11.77(7)$ & $4.54(23)$ & $6.68(15)$ & - & - & $5.50(16)$ \\
\hline 121. P. torta (Mart.) Radlk. & - & $0.91(54)$ & $2.70(32)$ & - & - & - \\
\hline 38. SOLANACEAE (1) & - & - & - & - & 0.61 & - \\
\hline 122. Solanum lycocarpum St. Hil. & - & - & - & - & $0.61(64)$ & - \\
\hline 39. STYRACACEAE (2) & 15.09 & 12.01 & 18.95 & 1.54 & 2.54 & 11.45 \\
\hline 123. Styrax camporium Pohl & - & - & - & - & - & $2.40(34)$ \\
\hline 124. Styrax ferrugineus Nees \& Mart. & $15.09(3)$ & $12.01(7)$ & $18.95(3)$ & $1.54(37)$ & $2.54(37)$ & $9.05(8)$ \\
\hline 40. SYMPLOCACEAE (3) & - & 3.11 & 0.54 & 1.61 & 0.78 & - \\
\hline 125. Symplococus nitens Benth. & - & - & - & $1.61(35)$ & - & - \\
\hline 126. S. rhamnifolia A.DC. & - & $1.52(49)$ & $0.54(56)$ & - & $0.78(56)$ & - \\
\hline 127. Symplocos sp. & - & $1.59(48)$ & - & - & - & - \\
\hline 41. VERBENACEAE (1) & 0.39 & - & - & 0.81 & 1.21 & - \\
\hline 128. Aegiphila lhotskyana Cham. & $0.39(71)$ & - & - & $0.81(49)$ & $1.21(49)$ & - \\
\hline
\end{tabular}


Cont. Tabela 1

\begin{tabular}{|c|c|c|c|c|c|c|}
\hline Famílias/espécies & $\begin{array}{l}\text { EEAI } \\
\text { DF }\end{array}$ & $\begin{array}{l}\text { APA } \\
\text { DF }\end{array}$ & $\begin{array}{l}\text { PNB } \\
\text { DF }\end{array}$ & $\begin{array}{l}\text { PAR } \\
\text { MG }\end{array}$ & $\begin{array}{l}\text { PAT } \\
\text { MG }\end{array}$ & $\begin{array}{l}\text { SIL } \\
\text { GO }\end{array}$ \\
\hline 42. VOCHYSIACEAE (8) & 38.69 & 51.68 & 37.93 & 32.65 & 37.53 & 78.50 \\
\hline 129. Qualea grandiflora Mart. & $8.59(10)$ & $15.98(3)$ & $18.16(4)$ & $11.84(5)$ & $26.89(1)$ & $39.29(1)$ \\
\hline 130. Q. multiflora Mart. & $1.44(47)$ & $5.31(19)$ & $1.06(50)$ & - & $3.89(28)$ & $1.75(45)$ \\
\hline 131. Q. parviflora Mart. & $19.78(2)$ & $19.29(2)$ & $15.75(5)$ & $8.08(11)$ & 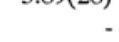 & $27.59(2)$ \\
\hline 132. Salveria convallariodora St. Hil. & $1.62(40)$ & - & - & $2.52(29)$ & - & $2.47(32)$ \\
\hline 133. Vochysia elliptica (Spr.) Mart. & $2.10(37)$ & $4.31(40)$ & $1.76(40)$ & $0.90(45)$ & $0.61(63)$ & $2.47(32)$ \\
\hline 134. Vochysia rufa (Spr.) Mart. & $5.16(20)$ & - & - & $9.31(9)$ & $5.00(0)$ & $7.40(10)$ \\
\hline 135. V. thysoidea Pohl & - & $6.59(13)$ & $1.30(47)$ & - & $5.52(18)$ & $1.40(10)$ \\
\hline 136. V. tucanorum (Spr.) Mart. & - & - & - & - & $0.62(61)$ & - \\
\hline NĀO IDENTIFICADAS & 0.42 & 0.77 & - & 0.69 & - & - \\
\hline 137. & - & $0.77(57)$ & - & - & - & - \\
\hline 138. & - & - & - & $0.69(58)$ & - & - \\
\hline 139. & $0.42(67)$ & - & - & - & - & - \\
\hline MORTAS & $14.90(4)$ & $15.64(4)$ & $28.25(1)$ & $17.48(3)$ & $14.34(4)$ & $13.08(4)$ \\
\hline
\end{tabular}

Fitossociologia

As estimativas da densidade por hectare foram de 1396 indivíduos para a Estação Ecológica de Águas Emendadas; 1394 indivíduos para APA Gama-Cabeça do Veado; 1036 indivíduos para o Parque Nacional de Brasília; 664 indivíduos para Paracatu; 981 indivíduos para Patrocinio e 1348 indivíduos paqra Silvânia.

As estimativas da área basal por hectare foram de 10,76 $\mathrm{m} 2$ para Estação Ecológica de Águas Emendadas; 10,64 m2 para a APA Gama-Cabeça do Veado; $8,32 \mathrm{~m} 2$ para o Parque Nacional de Brasília; 5,89 m2 para Paracatu; 5,79 m2 para Patrocínio e 11,30 m2 para Silvânia.

O percentual de árvores mortas foi 5,11\% para e Reserva Ecológica de Águas Emendadas; $5,67 \%$ para a APA Gama-Cabeça do Veado; $13,71 \%$ para o Parque Nacional de Brasília; 6,02\% para Paracatu; 5,20\% para Patrocínio e 6,10\% para Silvânia. Em geral, os valores estiveram próximos de 5\%, com exceção do Parque Nacional. A mais provável explicação foi a ocorrência recente de fogo na área.

Paracatu e Patrocínio se destacaram pela baixa densidade em número de indivíduos e em área basal em relação às demais áreas.

Na Tabela 1 são apresentadas as espécies amostradas em cada uma das localidades assim como seus valores e respectivas posições na classificação por ordem decrescente de IVI.

Entre as dez espécies masi importantes somente Qualea granfiflora esteve presente em todas as localidades.Qualea parviflora esteve entre as 10 mais importantes em quatro 
localidades amostradas, não sendo encontrada em Patrocínio e ocupando a $11^{\mathrm{a}}$ posição em Paracatu.

Styrax ferrugineus e Caryocar brasiliense destacaram-se em quatro localidades: Águas Emendades, APA Gama-Cabeça do Veado, Parque Nacional de Brasília e Silvânia.

Sclerolobium paniculatum destacou-se entre as 10 mais importantes na Estação Ecológica de Águas Emendadas, APA Gama-Cabeça de Veado e Paracatu. Ouratea hexasperma ocupou as primerias posições em importancia em três localidades, Estação Ecológica de Águas Emendadas, APA Gama-Cabeça de Veado e Parque Nacional de Brasília no Distrito Federal.

Dalbergia violacea ocupou as primeiras posições em importância em Patrocínio, Parque Nacional de Brasília e APA Gama-Cabeça do Veado. Kielmeyera coriacea destacou-se em Silvânia, Patrocínio e Estação Ecológica de águas Emendadas, enquanto que K. speciosa esteve entre as mais importantes em Estação Ecológica da Águas Emendadas e Parque Nacional de Brasília. Eugenia dysenterica, Roupala montana e Vochysia rufa estiveram entre as mais importantes em duas localidades, respectivamente Patrocínio e Paracatu. Parque Nacional de Brasília e Silvânia e Paracatu e Silvânia.

Dezesseis espécies ocuparam posição de destaque em pelo menos uma das diferentes localidades estudadas, são elas:Connarus suberosus, Teminalia argentea, Salacia crassifolia, Pouteris ramiflora, Tabebuia caraiba, Lafoensia pacari, Curatella americana, Piptocarpha rotundifolia, Byrsonima crassa, Didymopanax macrocarpum, Erythorxylum suberosum, Blepharocalyx salifolia, Xylopia sericea, Kielmeyera lathrophyton, Erythorxylum sp. e Bowdichia virgilioides. Destas espácies, 10 foram encontradas em Paracatu e Patrocínio (5 em cada área).

Algumas espécies alternaram suas posições em função da localidade, no Distrito Federal, Kielmeyera speciosa, que se destacou na Estação Ecológica de Águas Emendadas e no Parque Nacional de Brasília foi rara na APA Gama-Cabeça do Veado. Tabebuia caraiba foi rara na APA Gama-Cabeça do Veado e importante em Silvânia; Byrsonima coccolobifolia destacou-se no Parque Nacional de Brasília e apresentou baixa densidade em Paracatu. Essa tendência foi também contatada para Lafoensia pacari, Kielmeyra latrophyton, Blepharocalyx salicifolia e Bowdichia virgiloides. O caso mais extremo foi o da Curatella americana, primeira em IVI em Paracatu e ausente em todas as demais áreas com exceção de Estação Ecológica de Águas Emendadas onde foi rara.

Na Tabela 2 é apresentado um sumário com os principais parâmetros estruturais assim como as principais espécies de cada área.

Quanto às famílias, apenas Leguminosae e Vochysiaceae alcançaram valores altos em importância em todas as localidades. As famílias Annonaceae, Apocynaceae, Caryocaraceae, Compositae, Erythoxylaceae, Guttiferae, Malpighiaceae, Myrtaceae e Ochnaceae se destacaram em algumas localidades. Dentre as demais famílias as que tiveram presentes em todas as áreas e que sempre apresentaram valores baixos de IVI são: Araliaceae, Bignoniaceae, Connaraceae, Nyctaginaceae e Proteaceae. Labiatae, Moraceaew, Opiliaceae, Sapindaceae e Solanaceae foram amostradas em apenas uma das localidades com valores baixos para o IVI. 
Tabela 2 - Caracteristicas estruturais e as dez principais espécies encontradas no cerrado sensu stricto da Chapada Pratinha-Brasil

\begin{tabular}{|c|c|c|c|c|c|c|}
\hline Famílias/espécies & $\begin{array}{r}\text { EEAE } \\
\text { DF }\end{array}$ & $\begin{array}{r}\text { APA } \\
\text { DF }\end{array}$ & $\begin{array}{r}\text { PNB } \\
\text { DF }\end{array}$ & $\begin{array}{r}\text { PAR } \\
\text { MG }\end{array}$ & $\begin{array}{l}\text { PAT } \\
\text { MG }\end{array}$ & $\begin{array}{l}\text { SIL } \\
\text { GO }\end{array}$ \\
\hline $\begin{array}{l}\text { Número de espécies } \\
\text { Índice de Shannon } \mathrm{H}^{\prime} \\
\text { Densidade/ha } \\
\text { Área basal } \mathrm{m} 2 / \mathrm{ha} \\
\% \text { de árvores mortas em pé } \\
\text { Espécies mais importantes }\end{array}$ & $\begin{array}{r}72 \\
3,62 \\
1396 \\
10,76 \\
5,11 \\
\text { Scl pan } \\
\text { Qua par } \\
\text { Sty fer } \\
\text { Mortas } \\
\text { Kie cor } \\
\text { Car bra } \\
\text { Pou ram } \\
\text { Our hex } \\
\text { Kiel sp. } \\
\text { Qua gran }\end{array}$ & $\begin{array}{r}66 \\
3,56 \\
1394 \\
10,64 \\
5,67 \\
\text { Our hex } \\
\text { Qua par } \\
\text { Qua gra } \\
\text { Mortas } \\
\text { Car bra } \\
\text { Dal vio } \\
\text { Sty fer } \\
\text { Did mac } \\
\text { Scl pan } \\
\text { Byr cra }\end{array}$ & $\begin{array}{r}55 \\
3,34 \\
1036 \\
8,32 \\
13,71 \\
\text { Mortas } \\
\text { Our hex } \\
\text { Styr fer } \\
\text { Qua gra } \\
\text { Qua par } \\
\text { Car bras } \\
\text { Dal vio } \\
\text { Sal cra } \\
\text { Kie spe } \\
\text { Rou mon }\end{array}$ & $\begin{array}{r}60 \\
3,31 \\
664 \\
5,89 \\
6,02 \\
\text { Cur ame } \\
\text { Pip rot } \\
\text { Mortas } \\
\text { Eug dys } \\
\text { Qua gra } \\
\text { Ter arg } \\
\text { Con sub } \\
\text { Scl pan } \\
\text { Voc ruf } \\
\text { Bow vir }\end{array}$ & $\begin{array}{r}68 \\
3,53 \\
981 \\
5,79 \\
5,20 \\
\text { Qua gra } \\
\text { Eug dys } \\
\text { Ery sub } \\
\text { Mortas } \\
\text { Dal Vio } \\
\text { Ble sal } \\
\text { Kie cor } \\
\text { Xyl ser } \\
\text { Kie lat } \\
\text { Ery sp. }\end{array}$ & $\begin{array}{r}68 \\
3,31 \\
1348 \\
11,30 \\
6,10 \\
\text { Qua gra } \\
\text { Qua par } \\
\text { Kie cor } \\
\text { Mortas } \\
\text { Tab car } \\
\text { Laf pac } \\
\text { Car bra } \\
\text { Sty fer } \\
\text { Rou mon } \\
\text { Voc ruf }\end{array}$ \\
\hline
\end{tabular}

\footnotetext{
- Ble sal (Blepharocalyx salicifolia), Bow vir (Bowdichia virgilioides), Byr cra (Byrsonima crassa), Car bra (Caryocar brasiliense), Con sub (Connarus suberosus), Cur ame (Curatella americana), Dal vio (Dalbergia violacea), Dig mac (Didymopanax macrocarpum), Ery sp. (Erythroxylum sp.), Ery sub (Erythroxylum suberosum), Eug dys (Eugenia dysenterica), Kie lat (Kielmeyera lathrophyton), Kie cor (Kielmeyera coriacea), Kie spe (Kielmeyera speciosa), Kiel sp. (Kielmeyera sp.), Mortas em pé, Our hex (Ouratela hexasperma), Pip rot (Piptocarpha rotundifolia), Pou ram (Pouteria ramiflora), Qua par (Qualea parviflora), Qua gran (Qualea grandiflora), Rou mon (Roupala montana), Sal cra (Salacia crassifolia), Sel pan (Sclerolobium paniculatum), Sty fer (Styrax ferrugineus), Tab car (Tabebuia caraiba), Ter art (Terminalia argentea), Voc ruf (Vochysia rufa), Xyl ser (Xylopia sericea), Laf pac (Lafoensia pacari).
}

Estas diferenças indicam que as espécies do cerrado se caracterizam por se distribuirem espacialmente em "mosaicos", sempre com uma combinação de menos de 100 espécies por área estudada. Esta tendência pode ser observada em vários estudos realizados na região por outros autores como por exemplo, Ratter $e t$ al. 1973, Gibbs et al. 1983, Goodland 1979, Ratter 1986, 1987, Oliveira Filho et al. 1989. Mesmo em áreas próximas como as do Distrito Federal, aqui estudadas, as comunidades apresentaram-se florística e estruturalmente diferenciadas.

\section{Similaridade}

Para a avaliação da similaridade florística entre as diferentes áreas amostradas foram utilizados o índice de Sorensen (qualitativo) e o índice de Morisita (quantitativo) (Tabela 3).

$O$ índice de Sorensen, que avaliou apenas a presença ou ausência das espécies variou de 0,51 (APA Gama-Cabeça de Veado x Paracatu) até 0,77 (Parque Nacional de Brasília x APA Gama-Cabeça de Veado). Os menores valores de similaridade de Sorensen foram encontrados nas comparações com Patrocínio, 
onde todos os índices estiveram entre 0,51 e 0,59 , podendo então ser considerada a área com composição florística mais distinta.

Com a utilização do índice de Morisita, que considera a densidade das populações nas áreas amostradas, constatou-se uma redução na similaridade entre a maioria das áreas. Os valores encontrados para este índice variaram entre 0,21 (Paracatu x APA Gama-Cabeça do Veado) e 0,80 (APA Gama-Cabeça do Veado x Parque Nacional de Brasília). Este índice foi maior que o de Sorensen apenas na comparação entre a APA Gama-Cabeça de Veado e o Parque Nacional de Brasília.

Tabela 3 - Índices de similaridade das comunidades do cerrado sensu stricto da Chapada Pratinha, Brasil.

\begin{tabular}{lllllll}
\hline Área & EEAE & PNB & APA & SILV & PAR & PAT \\
\hline
\end{tabular}

\section{SORENSEN}

\begin{tabular}{|c|c|c|c|c|c|c|c|}
\hline $\mathrm{M}$ & EEAE & & 0.71 & 0.68 & 0.70 & 0.60 & 0.59 \\
\hline $\mathrm{O}$ & PNB & 0.71 & & 0.77 & 0.68 & 0.54 & 0.55 \\
\hline R & APA & 0.68 & 0.80 & & 0.69 & 0.51 & 0.57 \\
\hline S & SILV & 0.64 & 0.53 & 0.50 & & 0.61 & 0.53 \\
\hline & PAR & 0.24 & 0.27 & 0.21 & 0.21 & 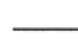 & 0.51 \\
\hline A & PAT & 0.38 & 0.43 & 0.42 & 0.49 & 0.28 & \\
\hline
\end{tabular}

As áreas amostradas apresentaram similaridade florística relativamente alta (Sorensen). Contudo, quando foram consideradas as densidades de ocorrência das espécies (Morisita) a similaridade só foi alta entre as três unidades de conservação do Distrito Federal e entre estas e SIlvânia-GO, indicando as diferênças estruturais da vegetação.

É interessante notar que os locais mais diferenciados foram Paracatu e Patrocínio, o que sugere a existência de gradientes latitudinais, longitudenais e altitudinais influenciando a estrutura e composição florística do cerrado s.s., mesmo em uma unidade fisiográfica relativamente homogênea e sob solos tipicamente distróficos, com nesta chapada. A presença de gradientes latitudinais e longitudinais foi constatada por Ratter \& Dargie (1992). A abundância de Curatella americana em Paracatu e sua raridade nas demais áres pode ser uma evidência da influência da altitude, uma vez que esta espécie costuma ser abundante em áreas de menor altitude tais como a baixada cuiabana em Mato Grosso e rara em maiores altitudes como no Distrito Federal (observação pessoal da primeira autora). 
No total foram encontradas pertencentes a 42 famílias; porém cada localidade apresentou uma diferente combinação de menos de 80 espécies e de aproximadamente 30 famílias.

A composição florística e a importancia fitossociológica das espécies variou para cada localidade, mesmo sendo a região fisiograficamente homogênia e sob solos tipicamente distróficos. Paracatu e Patrocínio, geograficamente mais distantes e situadas a menores altitudes em relação ao Distrito Federal foram as áreas mais diferenciadas.

Estes resultados sugerem que Paracatu e Patrocínio necessitam urgentemente da criação de unidades de conservação visando a proteção do patrimônio genético, principalmente levando-se em consideração a não disponibilidade de áreas extensas com vegetação nativa intocável, emfunção do grande desenvolvimento agrícola da região.

A realização de levantamentos florísticos e fitossociológicos baseados em zoneamentos fisionômicos e fisiográficos, como foi o caso deste trabalho, mostrase bastante recomendável quando do Planejamento de sistemas de unidades de conservação visando estabelecer reservas em áreas que abrajam a máxima diversidade florística e estrutural de uma determinada região.

\section{Agradecimentos}

À Secretaria de Planejamento da Presidência da Republica SEPLAN/ PROJETO PAMA pelo financiamento do Projeto. Ao CNPq pela concessão de bolsistas, aos colegas da Reserva Ecológica do IBGE, Jardim Botânico de Brasília e EMBRAPA que participaram das excursões de campo pela c olaboração e convivência, aos auxiliares de campo, estagiários e a todos que de alguma forma colaboraram para a realização do trabalho. Em especial ao Herbário da RECOR/IBGE pelo eficiênte processamento de todo material coletado.

\section{Referências bibliográficas}

Associação Brasileira do Produtores de Carvão Vegetal (ABRACAVE). 1988. Anuário Estatístico. Belo Horizonte-MG 11p.

COCHARANE, T. T., L.G.SANCHEZ, L.G. AZEVEDO, J.A. PORRAS, C.L. \& GARVER. 1985. Lands in tropical america, Cali, CIAT-EMBRAPA-CPAC, 3 vols.

DIAS, B.F. DE S. 1990. A conservação da natureza. In: Novaes Pinto, M. (org.) Cerrado:caracterização, ocupação e perspectivas. Brasília, Editora Universidade de Brasília. p.583-640.

EITEN, G. 1978. Delimitation of the cerrado concept. Vegetatio 36(3):169-178. -1984. Vegetation of Brasília. Phytocoenologia 12(2/3):271-292.

FREESE, F. 1962. Elementary florest sampling. USDA. 91p. 
FREESE, F. 1984. Vegetation of Brasília. Phytocoenologia. 12 (2/3): 271-292.

GIBBS, P.E.; LEITÃO FILHO, H.F \& SHEPHERD, G. 1983. Floristic composition and communiy struture in an area of cerrado in SE Brazil. Flora 173:433449.

GOODLAND, R. 1979. Analise Ecológica da vegetação do Cerrado. In: Ferri, M.G. (ed.) Ecologia do Cerrado (translated by Eugenio Amado). Belo Horizonte. Itatiaia. p. 61-160.

HORN, H. S. 1966 Measurement of "overlap" in comparative ecological studies. The American Naturalist 100(914):424.

MARGURRAN, A.E. 1988. Ecological diversity and its measurement. London. Chapman and Hall. 179p.

MUELLER-DOMBOIS, D. Y H. ELLENBERG, 1974. Aims and methods of vegetation ecology. New York, John Wiley \& Sons.

A. T. OLIVEIRA-FILHO; G.J. SHEPHERD; F.R. MARTINS \& W. H. STUBBLEBINE, 1989. Environmental factors affecting physiognomic and floristic variation in an area of cerrado in central Brazil. Journal of Tropical Ecology 5: 413-431.

RATTER, J.A. 1986. Notas sobre a vegetação da Fazenda Água Limpa. Editora UnB, textos universitários n. 003. 136p. Brasília.

RATTER, J.A. 1987. Notes on the vegetation of the Parque Nacional do Araguaia (Brazil). Notes of the Royal Botanic Garden of Edinburgh 44: 311-342.

RATTER, J.A. \& T.C.D. DARGIE. 1992. An analysis of the floristic composition of 26 cerrado areas in Brazil. Edin burgh Journal of Botany 49: 235-252.

RATTER, J.A.; P.W. RICHARDS, G. ARGENT \& D.R. GIFFORD, 1973. Observations on the vegetation of northeastern Mato Grosso. 1. The woody vegetation types of the Xavantina-Cachimbo expediton area. Phil. trans. of the Royal Society (b) 226: 449-492.

RIBEIRO, J.F.; S.M. SANO, J. MACEDO \& J.A. SILVA, 1983. Os principais tipos fisionômicos da região dos cerrados. Boletim de Pesquisa EMBRAPACPAC (21): 28p. 